\title{
Analysis of Synaptic Growth and Function in Drosophila with an Extended Larval Stage
}

\author{
Daniel L. Miller, ${ }^{1,2 *}$ Shannon L. Ballard, ${ }^{1 *}$ and Barry Ganetzky ${ }^{1}$ \\ ${ }^{1}$ Laboratory of Genetics and ${ }^{2}$ Institute on Aging, University of Wisconsin, Madison, Wisconsin 53706
}

The Drosophila larval neuromuscular junction (NMJ) is a powerful system for the genetic and molecular analysis of neuronal excitability, synaptic transmission, and synaptic development. However, its use for studying age-dependent processes, such as maintenance of neuronal viability and synaptic stability, are temporally limited by the onset of pupariation and metamorphosis. Here we characterize larval NMJ growth, growth regulation, structure, and function in a developmental variant with an extended third instar (ETI). RNAiknockdown of the prothoracicotropic hormone receptor, torso, in the ring gland of developing larvae leaves the timing of first and second instar molts largely unchanged, but triples duration of the third instar from 3 to $9.5 \mathrm{~d}$ (McBrayer et al., 2007; Rewitz et al., 2009). During this ETI period, NMJs undergo additional growth (adding $>50$ boutons/NMJ), and this growth remains under the control of the canonical regulators Highwire and the TGF $\beta$ /BMP pathway. NMJ growth during the ETI period occurs via addition of new branches, satellite boutons, and interstitial boutons, and continues even after muscle growth levels off. Throughout the ETI, organization of synapses and active zones remains normal, and synaptic transmission is unchanged. These results establish the ETI larval system as a viable model for studying motor neuron diseases and for investigating time-dependent effects of perturbations that impair mechanisms of neuroprotection, synaptic maintenance, and response to neural injury.

\section{Introduction}

The Drosophila larval neuromuscular junction (NMJ) is a powerful model system for uncovering and characterizing genetic and molecular mechanisms that regulate synaptic growth, structure, and function. The NMJ offers advantageous features for neurogenetic analyses including a segmentally repeated and stereotypic morphology, which allows easy quantification of morphological and functional properties (Packard et al., 2003; Ruiz-Cañada and Budnik, 2006; Collins and DiAntonio, 2007). In addition, the molecular mechanisms that regulate synapse formation and function are inherently similar between vertebrates and Drosophila (Keshishian et al., 1996; Featherstone and Broadie, 2000). Previous studies have uncovered key molecules and processes that govern NMJ development including TGF $\beta / B M P$ and Wnt/Wg pathways, endocytic machinery, autophagy, and electrical excitability (Budnik et al., 1990; Keshishian et al., 1996; Featherstone and Broadie, 2000; Marqués,

\footnotetext{
Received Jan. 31, 2012; revised Aug. 8, 2012; accepted Aug. 13, 2012

Author contributions: D.L.M., S.L.B., and B.G. designed research; D.L.M. and S.L.B. performed research; D.L.M. and S.L.B. analyzed data; D.L.M., S.L.B., and B.G. wrote the paper.

This work was supported by National Institutes of Health Grants T32 AG000213 (D.L.M.), F32 NS067843 (S.L.B.), R21 NS078342, R01 NS015390, and R01 AG033620 (B.G.).We would like to especially thank Dr. Michael O'Connor for his helpful and stimulating discussions and for very generously sharing stocks with us. We also thank K. Luedke, A. Arthur, C. Kuchenbecker, and C. Hannon for their technical assistance. Anti-Dlg and anti-Brp were obtained from the Developmental Studies Hybridoma Bank developed under the auspices of the National Institutes of Health-National Institute of Child Health and Human Development and maintained by The University of lowa, Department of Biology, lowa City, IA 52242

${ }^{*}$ D.L.M. and S.L.B. contributed equally to this work.

The authors declare no competing financial interests.

Correspondence should be addressed to Barry Ganetzky, 425 Henry Mall, University of Wisconsin—Madison, Madison, WI 53706. E-mail: ganetzky@wisc.edu.

DOI:10.1523/JNEUROSCI.0508-12.2012

Copyright $\odot 2012$ the authors $\quad 0270-6474 / 12 / 3213776-11 \$ 15.00 / 0$
}

2005; Dickman et al., 2006; Collins and DiAntonio, 2007; Shen and Ganetzky, 2009).

Despite these advantages, the short duration of the third instar stage limits the use of the larval NMJ as a model system for timedependent studies. Because the time interval between hatching and pupariation in D. melanogaster is $<1$ week, the larval NMJ is not well suited for studying biological mechanisms, such as neurodegeneration, that generally occur over longer time intervals. In principle, this constraint could be overcome if the larval stage were substantially extended without causing significant perturbations of NMJ structure and function. The mechanisms that maintain NMJ structure over time, how synapses are compromised with age or disease, and long-term effects of neuronal injury could then be investigated in these larvae.

In Drosophila, the steroid hormone 20-hydroxyecdysone (20HE) mediates the proper timing of larval molts and metamorphosis. The neuropeptide prothoracicotropic hormone (PTTH) acts on the prothoracic gland (PG) to produce and release the 20HE precursor, ecdysone (Gilbert et al., 2002). Recent studies showed that ablation of PTTH-producing neurons (McBrayer et al., 2007) or knockdown of the PTTH receptor, torso, in the PG (phm-Gal4>UAS-torso-RNAi) (Rewitz et al., 2009) leads to a tripling of the third instar larval stage from $3 \mathrm{~d}$ in control larvae to over 9 d. phm-Gal4>UAS-torso-RNAi larvae develop normally, continue to grow in size during the extended third instar (ETI) stage, undergo pupariation, and eclose as larger adult flies (Rewitz et al., 2009).

Thus, the NMJ of phm-Gal4>UAS-torso-RNAi larvae could provide an excellent background for studying age-dependent mechanisms. However, it is necessary to first characterize the basic properties of NMJs during the extended period of larval 
development. To this end, we examined NMJ growth, structure, and function in phm-Gal4>UAS-torso-RNAi larvae. Here we show that during the ETI period, NMJs continue to grow by addition of new boutons, and that this growth is dependent upon known regulators of NMJ development. In addition, the integrity and structure of the NMJ remains stable, and normal synaptic transmission is maintained. Thus, the ETI stage in phmGal4>UAS-torso-RNAi larvae provides a novel and valuable framework for experiments that probe time-dependent neurobiological processes while taking advantage of all the powerful features of the larval NMJ.

\section{Materials and Methods}

Fly stocks. $w^{1118}$ was used as a wild-type control for genetic background, and experiments were performed in a $w^{1118}$ background. phm-Gal4 and UAStorso-RNAi (Rewitz et al., 2009) were provided by M. O'Connor (University of Minnesota, Minneapolis, MN) and Vienna Drosophila RNAi Center (\#36280), respectively. hiw ${ }^{N D 8}$ (Wan et al., 2000) was provided by A. DiAntonio (Washington University, St. Louis, MO). BG380-Gal4 was provided by V. Budnik (1996). The following stocks were obtained from the Bloomington Stock Center: wit ${ }^{B 11}$ (Marqués et al., 2002), UAS-EcRA-RNAi, UASEcRB1-RNAi, UAS-EcRC(97)-RNAi, and 24B-Gal4.

Developmental timing of larvae. Eggs were laid on apple juice agar plates for $12 \mathrm{~h}$ at $25^{\circ} \mathrm{C}$. Newly hatched first instar larvae were collected $36 \mathrm{~h}$ after egg lay (AEL), thus $0-12 \mathrm{~h}$ after hatching. Larvae were placed in softened standard molasses food on apple juice plates and raised at $25^{\circ} \mathrm{C}$. Every $36 \mathrm{~h}$, the larvae were transferred onto fresh molasses food/apple juice plates to avoid desiccation. Larvae were collected at designated time points, placed in $\mathrm{Ca}^{2+}$-free saline, and dissected for analysis.

Immunohistochemistry. Female larvae from designated time points were dissected in $\mathrm{Ca}^{2+}$-free saline and fixed in $4 \%$ paraformaldehyde in PBS for $20 \mathrm{~min}$ unless otherwise noted. Larval body walls were incubated in primary and secondary antibodies overnight at $4^{\circ} \mathrm{C}$ while rocking. They were then mounted in VectaShield (Vector Laboratories) for microscopic analysis. The following antibodies were used: fluorescein isothiocyanate (FITC)-conjugated anti-horseradish peroxidase (HRP) at 1:100 (Jackson ImmunoResearch), mouse anti-Dlg at 1:1000 (Developmental Hybridoma Studies Bank), mouse anti-nc82 (Bruchpilot) at 1:250 (Developmental Hybridoma Studies Bank), and anti-DvGlut 1:5000 (generous gift from A. DiAntonio). For use of anti-GluRIII (1:5000) (A. DiAntonio), larvae were fixed in Bouin's fixative for $8 \mathrm{~min}$, followed by antibody incubation as above. Species-specific Alexa 405, Alexa 488, Alexa 568, and Alexa 633 (Invitrogen) secondary antibodies were used at 1:200.

Imaging and quantification. Quantification of bouton number was performed at NMJ4 due to its relative simplicity. However, comparable phenotypes were observed at other NMJs. Segments A2-A4 were analyzed for bouton number and muscle area. At least $25 \mathrm{NMJs}$ of each genotype were analyzed for each time point. Confocal images were obtained on an LSM 510 confocal microscope (Carl Zeiss) with PlanApochromat $63 \times$ NA 1.4 oil differential interference contrast objectives and accompanying software. Images were processed in Image (National Institutes of Health) and Adobe Photoshop software. Muscle area was determined using the draw function of Zeiss AIM software on live DIC images generated on an Axiomager Z1 (Carl Zeiss). Branch points were defined as any branch of two or more boutons off of the primary nerve terminal and any subsequent branches off of these secondary branches. Branch length was determined using ImageJ, where arbors of primary and secondary branch nerve terminals were measured starting at the first bouton or branch point after defasciculation (whichever occurred first). For quantification, we defined a bouton as a synaptic swelling compared with neighboring axonal segments that were labeled with the presynaptic marker, $\alpha$-HRP, and with the postsynaptic marker, $\alpha$-Dlg. Boutons were quantified directly from immune-stained preparation under a confocal microscope, which afforded better resolution of boutons through the $Z$-plane and enabled visualization of boutons that were not always evident in a photographic image. Satellite boutons were defined as extensions of two or fewer boutons off of the nerve branches. Bouton density was measured by averaging the total number of boutons within the first $20 \mu \mathrm{m}$ and the terminal $20 \mu \mathrm{m}$ of primary or secondary branches.

Electrophysiology. Electrophysiology was performed on muscle 6 in segments A3-A5 of larvae at designated time points using standard techniques (Jan and Jan, 1976). Dissections were performed in HL3 saline containing $0.4 \mathrm{~mm} \mathrm{Ca}^{2+}$, and intracellular recordings were performed in HL3 containing the indicated $\mathrm{Ca}^{2+}$ concentration. Recording electrodes (resistance: 15-20 $\mathrm{M} \Omega$ were filled with $3 \mathrm{M} \mathrm{KCl}$ and stimulating electrodes with saline. Undamaged muscles with a minimum resting potential of $60 \mathrm{mV}$ and input resistance of $5 \mathrm{M} \Omega$, were selected for recording (no significant difference in either parameter was observed across all genotypes and time points assayed). Recordings were acquired using an AxoClamp 2B amplifier, digitized with a Molecular Devices Digidata 1440A digitizer, amplified with a Brownlee Precision 410 amplifier, and recorded using pClamp10.3 software (Molecular Devices). Mean excitatory junction potential ( $\mathrm{mEJP}$ ) amplitudes were calculated from 75 consecutive traces (26-100 of 100 stimulations). Average mEJP amplitude and frequency were determined using Mini Analysis Software v 6.0.7 (Synaptosoft) by averaging 70 consecutive events for each synapse. Quantal content was determined by dividing average EJP amplitude of a synapse by the average $\mathrm{mEJP}$ amplitude from the same synapse. For this calculation EJP amplitudes were corrected for nonlinear summation according to McLachlan and Martin (1981).

Statistical analyses. Error bars indicate SEM, and Student's $t$ test was performed for all statistical analyses. We report the significance values to be $<0.01$ or 0.001 denoted by one or two stars, respectively.

\section{Results}

Characterization of NMJ growth in larvae with an ETI stage

Although wild-type larvae typically spend only $3 \mathrm{~d}$ in the third larval instar at $25^{\circ} \mathrm{C}$ before undergoing pupariation, this period can be greatly expanded by genetic manipulation of the hormonal mechanism that regulates larval development. The secreted PTTH triggers production and release of the steroid hormone ecdysone, which regulates progression of larval development. Larvae with reduced transcript levels of the receptor tyrosine kinase torso, the receptor for PTTH (Rewitz et al., 2009), in the PG of the ring gland (phm-Gal4>UAS-torso-RNAi), remain in the third larval instar for up to $9 \mathrm{~d}$ (Fig. $1 P$; Rewitz et al., 2009). During this ETI stage, larval body size continues to increase. This extension of larval development and enhanced larval growth raises interesting questions about synaptic development. Do larval NMJs stop growing after they reach the maximum size they would achieve during normal larval growth or do they retain sufficient plasticity to continue to grow? If the latter, is NMJ growth coordinated with the increase in muscle size during the extended larval phase? Answers to these questions should reveal important new insights about regulation of NMJ growth not obtainable from studies of wild-type larvae. To address these questions, we examined NMJ morphology of third instar larvae at defined time points AEL, up to the time of pupariation. As one parameter of NMJ growth, we counted the number of boutons per NMJ on muscle 4 (NMJ4). During the third instar, bouton number in $w^{1118}$ control larvae increased $~ 35 \%$ from $84 \mathrm{~h}$ AEL $(14.3 \pm 0.5)$ to pupariation at $144 \mathrm{~h}$ AEL $(19.3 \pm 0.7)$ (Fig. $1 A-C, E-G, I-K, P)$. NMJs of phm-Gal4/+ and UAS-torso$R N A i /+$ larvae grew from $17.1 \pm 0.6$ to $24.9 \pm 0.8$ boutons $(46 \%$ increase) and $15.7 \pm 0.4$ to $23.9 \pm 1.0$ boutons ( $52 \%$ increase), respectively. Previous studies have suggested that this growth is correlated with an increase in the muscle surface area during the same time interval (Guan et al., 1996). Our data are consistent with this idea. In control larvae, the increase in surface area for muscle 4 parallels the increase in bouton number. From 84 to $144 \mathrm{~h}$ AEL, muscle area in $w^{1118}$ larvae increased by $48 \%$, in phm-Gal4/+ larvae by $85 \%$, and in UAS-torso-RNAi/+ larvae by 

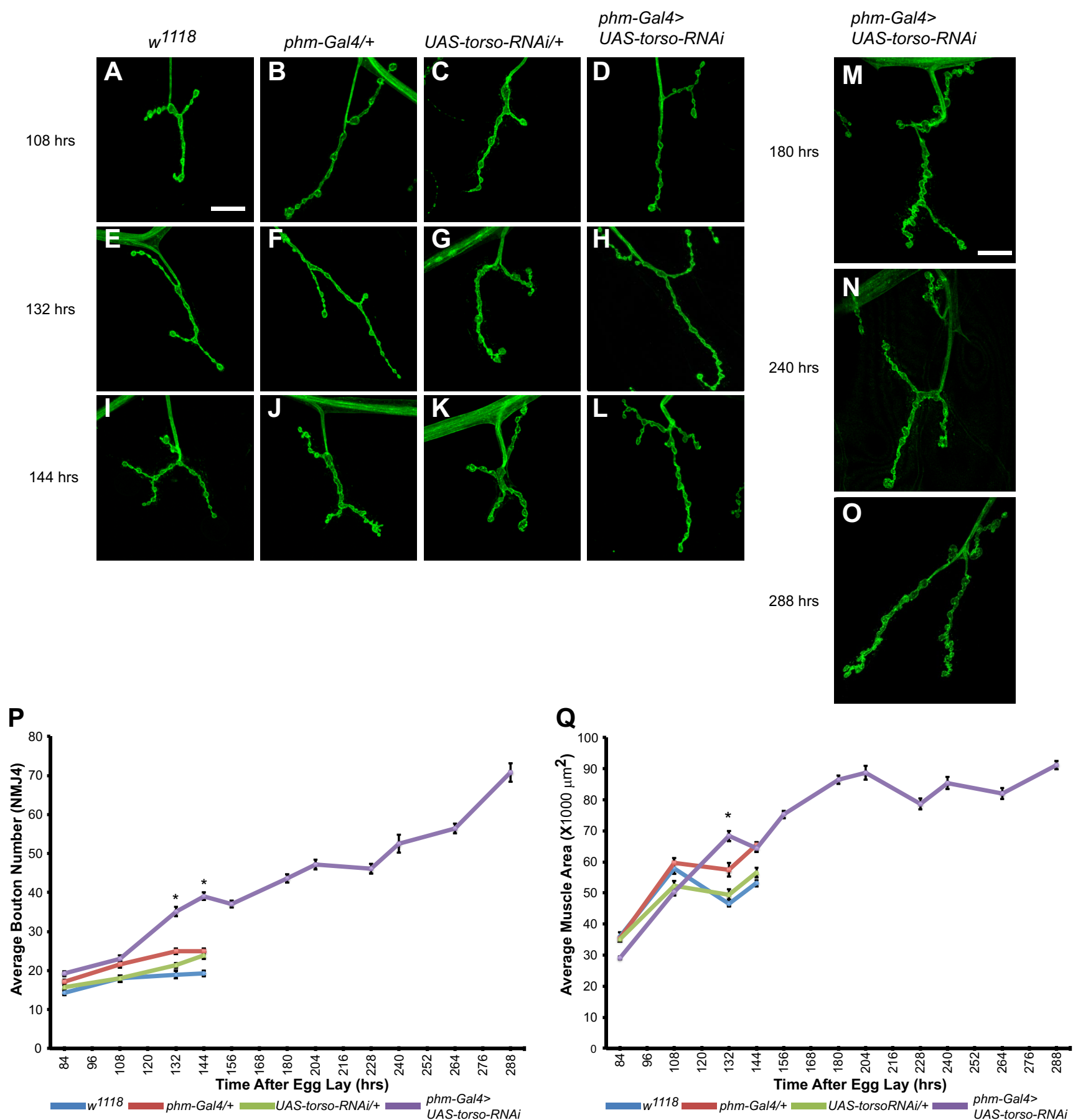

Figure 1. Synapse and muscle growth continues during extended larval period. A-O, Confocal images of NMJ4 labeled with FITC-anti-HRP. P, Quantification of average bouton number for $w^{1118}$ (blue line), phm-Gal4/+ (red line), UAS-torso-RNAi/+ (green line), and phm-Gal4>UAS-torso-RNAi (purple line) larvae throughout the third instar. $\mathbf{Q}$, Average muscle area of control and phm-Gal4 > UAS-torso-RNAi larvae. Control (A-C) and phm-Gal4>UAS-torso-RNAi (D) larvae display no significant differences in bouton number at $108 \mathrm{~h}$ AEL (P). phm-Gal4 $>$ UAS-torso-RNAi larvae display an increase in bouton number compared with control larvae at 132 and $144 \mathrm{~h} \mathrm{AEL}(\boldsymbol{E}-\mathbf{L}, \boldsymbol{P})$. Bouton number and muscle area continue to increase between 144 and $288 \mathrm{~h}$ AEL $(\boldsymbol{M}-\boldsymbol{Q})$. Scale bar, $20 \mu \mathrm{m}$. Error bars represent SEM. ${ }^{*} p<0.01$.

$62 \%$ (Fig. 1Q). Although the overall rates of growth are greater (see below), we observe a similar parallel increase in phmGal4>UAS-torso-RNAi larvae, where muscle area and NMJ size increase by $120 \%$ and $100 \%$, respectively, between 84 and $144 \mathrm{~h}$ AEL (Fig. 1D, $H, L-Q)$ ). Together, these results support the idea that as larvae progress through the third instar stage, NMJ growth parallels the increase in muscle size.

At early time points of the third instar, NMJ growth in phmGal4>UAS-torso-RNAi larvae is similar to control larvae. How- ever, at 132 and $144 \mathrm{~h}$ AEL, phm-Gal4>UAS-torso-RNAi larvae display a significant increase in bouton number compared with control larvae (e.g., $24.9 \pm 0.8$ for phm-Gal4/+ vs $39.1 \pm 1.0$ for phm-Gal4>UAS-torso-RNAi at $144 \mathrm{~h} \mathrm{AEL).} \mathrm{This} \mathrm{augmentation}$ of synaptic growth might be explained by a concomitant increase in muscle area. Indeed, phm-Gal4>UAS-torso-RNAi larvae exhibit a significant expansion of muscle area at $132 \mathrm{~h} \mathrm{AEL;} \mathrm{how-}$ ever, by $144 \mathrm{~h}$ AEL, phm-Gal4>UAS-torso-RNAi larval muscle area does not differ from control larvae (Fig. 1Q). Thus, an in- 
crease in muscle area might be associated with the initial addition of boutons at $132 \mathrm{~h} \mathrm{AEL}$, but it cannot account for the further increase in bouton number at $144 \mathrm{~h}$ AEL in phm-Gal4>UAStorso-RNAi larvae even before the onset of extended larval development.

\section{Presynaptic ecdysone signaling influences NMJ growth}

To examine factors other than muscle size that could affect the increase in bouton number at 132 and $144 \mathrm{~h} \mathrm{AEL} \mathrm{in} \mathrm{phm-}$ Gal4 $>$ UAS-torso-RNAi larvae, we asked whether a reduction in torso mRNA levels in presynaptic or postsynaptic cells influences NMJ growth. Although expression of phm-Gal4 has been observed only in the PG (Rewitz et al., 2009; data not shown), the possibility remains that torso-RNAi expression outside the PG could influence NMJ growth. We used the muscle-specific $24 B$ Gal4 and the neuron-specific BG380-Gal4 drivers to reduce the levels of torso either postsynaptically or presynaptically, respectively. Reduction of torso mRNA in either muscles or neurons does not affect NMJ growth (Fig. 2A). Because the ecdysone precursor is secreted from the ring gland and affects growth and development of distant tissues throughout the entire larva, we tested whether reduction in ecdysone signaling at the NMJ is associated with the observed increase in bouton number at 132 and $144 \mathrm{~h}$ AEL in phm-Gal4>UAS-torso-RNAi larvae compared with controls. There are three characterized isoforms of the ecdysone receptor (EcR): EcRA, EcRB1, and EcRB2. We used BG380-Gal4 and 24B-Gal4 to drive expression of isoformspecific RNAi to reduce levels of EcRA or EcRB1 isoforms either presynaptically or postsynaptically. We also used an RNAi construct against a common region of all EcR isoforms (UAS-EcRC$R N A i)$ to decrease ecdysone signaling at the NMJ. Reduction of ecdysone receptors postsynaptically does not affect bouton number either at 120 or $132 \mathrm{~h}$ AEL (Fig. $2 \mathrm{~B}$; data not shown). However, presynaptic expression of any of the three EcR RNAi constructs results in a significant increase in bouton number compared with control larvae at $120 \mathrm{~h}$ AEL (Fig. 2 B). This increase in NMJ growth is also observed at $132 \mathrm{~h}$ AEL in BG380Gal4>UAS-EcRA-RNAi and BG380>UAS-EcRC-RNAi larvae (data not shown). The increase in bouton number associated with presynaptic reduction of ecdysone signaling is accompanied by an increase in muscle area (Fig. 2C; data not shown). These results suggest that ecdysone signaling normally functions in motor neurons to restrict NMJ growth and that a reduction in systemic ecdysone titers could promote an early increase in bouton number in phm-Gal4>UAS-torso-RNAi larvae even before the onset of extended larval development. Furthermore, the increase in muscle size associated with presynaptic reduction in ecdysone signaling suggests some type of trans-synaptic signaling mechanism by which muscle size also increases as the presynaptic terminal expands.

\section{NMJs continue to grow during the ETI period}

NMJ growth in control larvae terminates with the onset of pupariation $\sim 144 \mathrm{~h} \mathrm{AEL}$ and the subsequent remodeling of the nervous system during metamorphosis. Consequently, one might expect that the mechanisms regulating larval NMJ growth would be selected by evolution to operate only over the normal time interval of the larval stage. However, in phm-Gal4>UAStorso-RNA $i$ larvae, the third larval instar continues for up to 6 more days beyond the usual onset of pupariation. During this time, the larvae continue to grow, resulting in the production of large pupae (Rewitz et al., 2009). Thus, it is of interest to determine whether NMJs in ETI larvae terminate growth after reach- ing the maximum size for control larvae or if they continue to grow throughout the ETI. To investigate this question, we assessed NMJ growth in phm-Gal4>UAS-torso-RNAi larvae at various time points up to the onset of pupariation at $288 \mathrm{~h}$ AEL. Between $144 \mathrm{~h}$ (onset of pupariation in control larvae) and $288 \mathrm{~h}$ AEL, the number of boutons continues to increase steadily, reaching $70.8 \pm 2.4$ boutons, an $80 \%$ increase over the bouton count at $144 \mathrm{~h} \mathrm{AEL} \mathrm{(39.1} \pm$ 1.0). Until 180-204 h AEL, the increase in bouton numbers is paralleled by an increase in muscle growth. However, although NMJ growth continues after $204 \mathrm{~h}$ AEL, muscle area remains relatively constant thereafter (Fig. 1, $M-Q)$. These results demonstrate that NMJs, as measured by an increase in bouton number, maintain continuous growth throughout the extended third larval instar in phm-Gal4>UAStorso- $R N A i$ larvae indicating that there is no inherent time constraint for NMJ growth and that NMJ growth does not terminate upon reaching a certain size. Moreover, only a portion of the overall NMJ growth in phm-Gal4>UAS-torso-RNAi larvae occurs in concert with an increase in muscle size. Thus, although NMJ growth and muscle growth usually occur in parallel, they are not necessarily mechanistically coupled and cues other than an increase in muscle size can stimulate NMJ expansion.

To characterize the increase in NMJ growth during the ETI period in phm-Gal4>UAS-torso-RNAi larvae in greater detail, we quantified several other morphological parameters in addition to bouton number. One such parameter is the number of branch points per NMJ4. In control larvae, we do not observe any increase in the number of branch points between 84 and $144 \mathrm{~h} \mathrm{AEL}$ (data not shown) consistent with the observations of Zito et al. (1999). At 144 h AEL, phm-Gal4>UAS-torso-RNAilarvae display a small, but significant increase in branch number compared with control larvae at the same time point $(2.3 \pm 0.2$ for UAS-torso$R N A i /+$ vs $3.3 \pm 0.2$ for phm-Gal4>UAS-torso-RNAi) (Fig. $3 A$ ) or with phm-Gal4>UAS-torso-RNAi larvae at $84 \mathrm{~h}$ AEL (data not shown). Between 144 and $288 \mathrm{~h} \mathrm{AEL}$, in phm-Gal4>UAS-torso$R N A i$ larvae the number of branch points per NMJ4 increases further to $4.3 \pm 0.3$ (Fig. $3 A$ ).

We also examined the average length of the NMJ terminal during the ETI by summing the lengths of all primary and secondary branches for each NMJ4 (see Materials and Methods). At $144 \mathrm{~h} \mathrm{AEL}$, the average terminal length in phm-Gal4>UAS-torso$R N A i$ larvae is larger than in control larvae $(133.3 \pm 6.7 \mu \mathrm{m}$ for $U A S$-torso-RNAi/+ vs $185.9 \pm 12.2 \mu \mathrm{m}$ for $p h m-G a l 4>U A S$ torso-RNAi) (Fig. $3 B$ ). Despite the significant increase in bouton number in phm-Gal4>UAS-torso-RNAi larvae between 144 and $288 \mathrm{~h}$ AEL, the terminal length does not change significantly $(185.9 \pm 12.2 \mu \mathrm{m}$ for $144 \mathrm{~h}$ vs $215.8 \pm 010.5 \mu \mathrm{m}$ for $288 \mathrm{~h}$ ) (Fig. $3 B$ ) resulting in an increase in the relative density of boutons per unit length. We quantified this parameter by determining the average number of boutons within a span of $20 \mu \mathrm{m}$ along the primary and secondary branches of NMJ4 (see Materials and Methods). Bouton density at $144 \mathrm{~h}$ AEL does not differ between control and phm-Gal4>UAS-torso-RNAi larvae (Fig. 3C). However, between 144 and $288 \mathrm{~h}$ AEL, there is a significant increase in bouton density in phm-Gal4>UAS-torso-RNAi larvae $(6.0 \pm 0.3$ vs $8.2 \pm 0.4$ ) (Fig. $3 C$ ). Finally, we quantified the number of satellite boutons (e.g., small boutons budding off from boutons on the main synaptic axis). In control larvae, there are very few satellite boutons $(<2 / \mathrm{NMJ} 4)$, and this number does not increase between 84 and $144 \mathrm{~h}$ AEL (data not shown). However, in phmGal4>UAS-torso-RNAi larvae, satellite boutons are added steadily throughout the ETI period, reaching a total of $11.6 \pm 1.0$ 
A
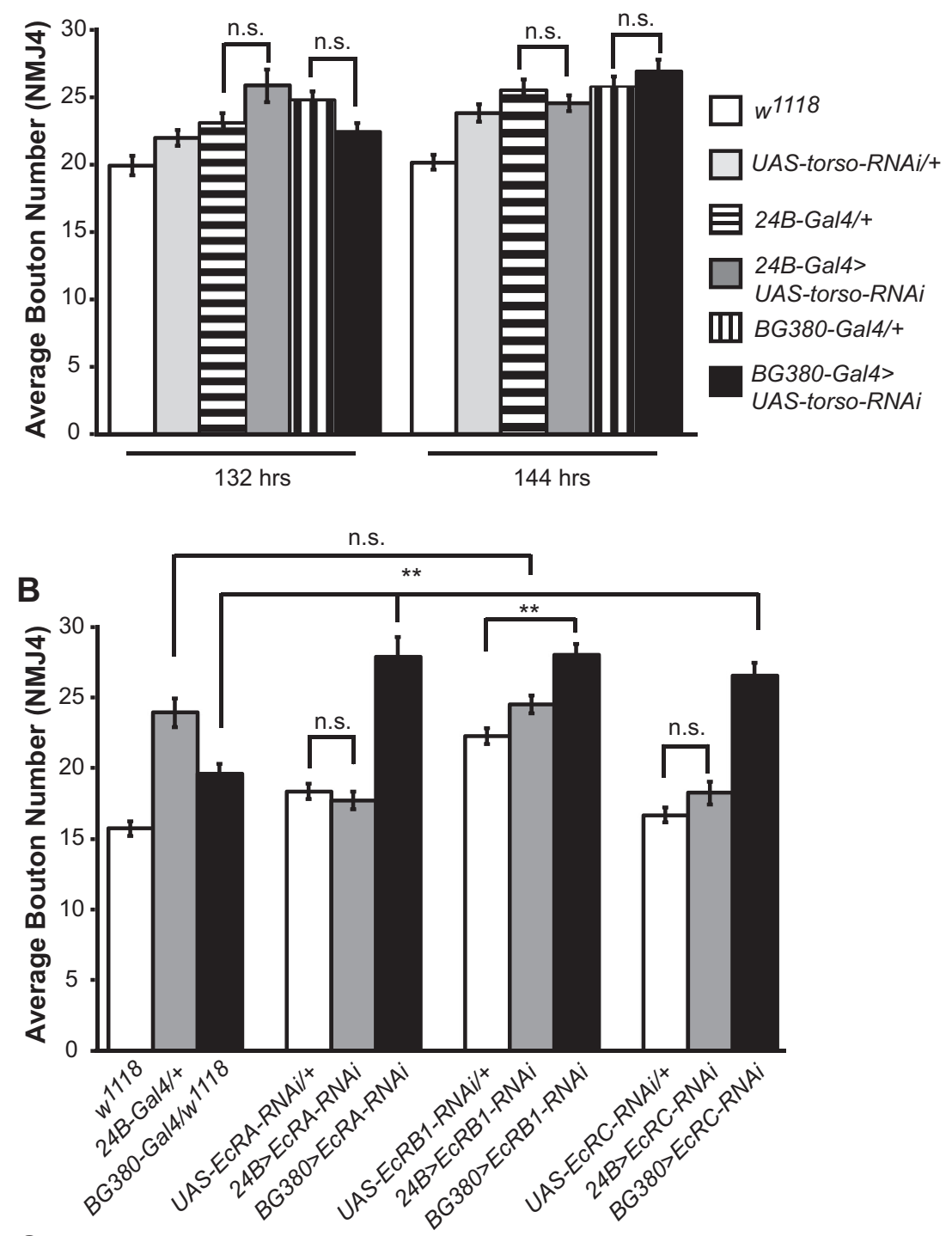

c

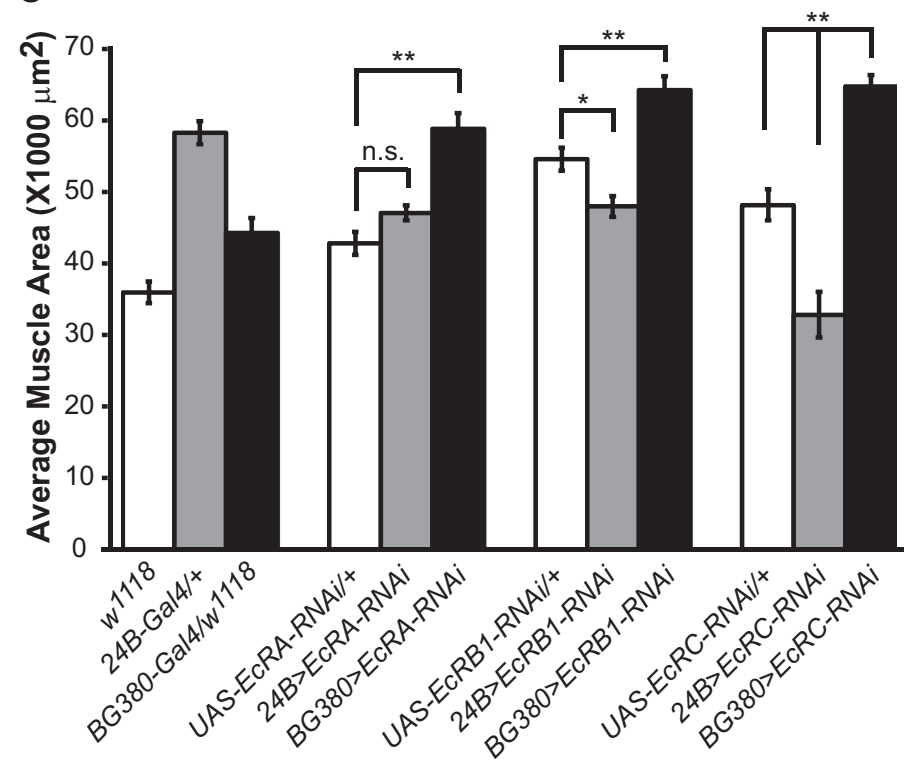

Figure 2. Synaptic growth is negatively regulated by presynaptic ecdysone signaling. A, Quantification of bouton number at NMJ4 reveals that reduction of torso mRNA levels using RNAi (UAS-torso-RNAi) in either the muscle (24B-Ga/4) or the motor neuron at $288 \mathrm{~h}$ AEL, a $180 \%$ increase compared with 144 h AEL $(4.1 \pm 0.5)$ (Fig. 3D).

Thus, the increase in bouton number at NMJ4 during the ETI in phm-Gal4>UAStorso-RNAi larvae appears to involve several distinct growth mechanisms. First, there is an increase in the total number of synaptic branches. Second, although these branches do not increase in length they continue to add new boutons interstitially resulting in an increase in bouton density per unit length. Finally, budding of new boutons from pre-existing boutons continues throughout the ETI generating a sizeable increase in the number of satellite boutons.

NMJ growth in phm-Gal4>UAS-torso$R N A i$ larvae during ETI is under the control of known growth regulators Does the continued growth of NMJ4 in phm-Gal4>UAS-torso-RNAi larvae during the ETI depend on the activities of the same positive and negative regulators of NMJ growth that are known to operate earlier during normal larval development or are entirely new mechanisms engaged? To address this question, we focused on the effects of two key regulatory genes: highwire ( $h i w$ ), which encodes an E3 ubiquitin ligase that is one of the strongest known negative regulators of NMJ growth (Wan et al., 2000), and wishful thinking (wit), which encodes a type II BMP receptor for the ligand encoded by glass bottom boat $(g b b)$, a potent positive regulator of NMJ growth (Aberle et al., 2002; Marqués et al., 2002). We found that NMJ growth during the ETI period is not only responsive to these regulators, but shows an enhanced sensitivity to their dosage compared with developmentally normal larvae.

We tested the effect of heterozygosity for hiw on NMJ growth in phm-Gal4> $U A S$-torso-RNAi larvae. Between 84 and 144 h AEL, phm-Gal4>UAS-torso-RNAi larvae heterozygous for hiw (hiw ${ }^{N D 8} /+$; phm-Gal4>UAS-torso-RNAi) exhibit no significant changes in bouton number at NMJ4 compared with $+/+$; phmGal4>UAS-torso-RNAi larvae (39.1 \pm 1.0 vs $36.3 \pm 1.3$ boutons, respectively) (Fig. $4 A, B, M)$. At $156 \mathrm{~h}$ AEL, $12 \mathrm{~h}$ after control

(BG380-Gal4) does not affect synaptic growth. B, C, Quantification of bouton number $(\boldsymbol{B})$ and muscle area $(\boldsymbol{C})$ at NMJ4 in larvae with reduced ecdysone receptor isoform levels (UASECRA-RNAi, UAS-ECRB1-RNAi, or UAS-ECRC-RNAi) at $120 \mathrm{~h}$ AEL. Loss of ecdysone signaling in the motor neuron leads to an increase in bouton number and muscle area compared with control larvae. Error bars represent SEM. ${ }^{*} p<0.01,{ }^{* *} p<$ $0.001 ;$ n.s. $=$ not statistically significant. 
A

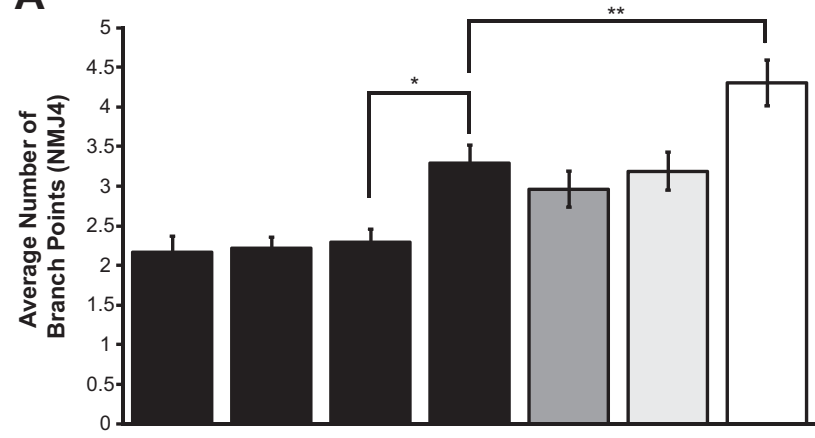

B

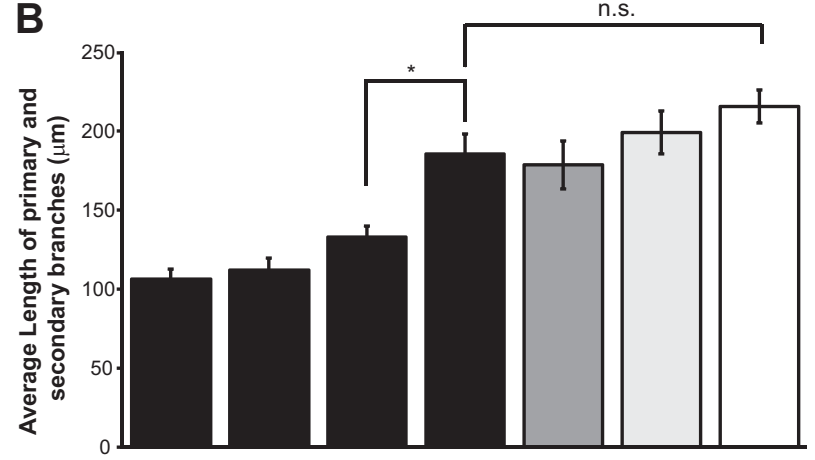

C

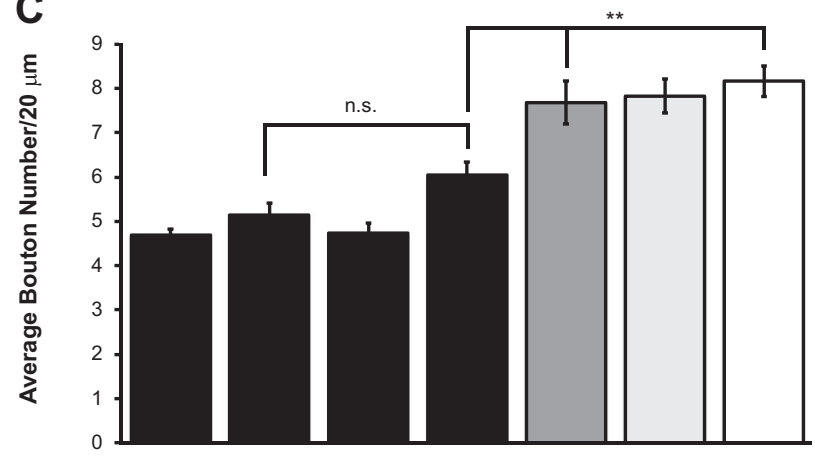

D

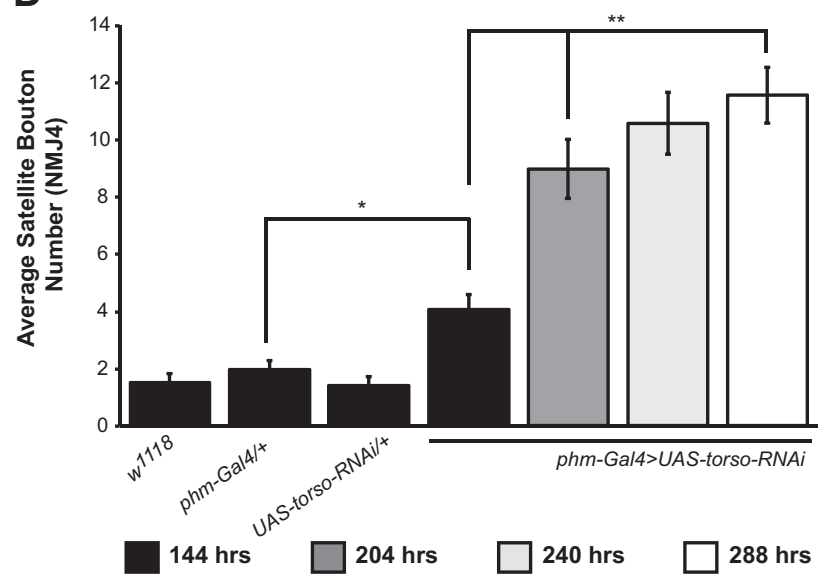

Figure 3. Characterization of synaptic morphological features during the extended larval period. A, Quantification of average branching points at NMJ4. B, Average length of primary and secondary branches at NMJ4. C, Average number of boutons per $20 \mu \mathrm{m}$ along NMJ4 branches. D, Quantification of average satellite bouton number at NMJ4. phm-Gal4>UAS-torso-RNAi larvae exhibit an increase in average branch number, bouton density along the nerve branches, and average satellite bouton number at $\operatorname{NMJ4}(\boldsymbol{A}, \boldsymbol{C}, \boldsymbol{D})$. Error bars represent $\mathrm{SEM}$. ${ }^{*} p<0.01$, ${ }^{* *} p<0.001 ;$ n.s. $=$ not statistically significant. larvae pupariate, $h i w^{N D 8} /+$; phm-Gal4>UAS-torso-RNAi larvae exhibit a significant increase in bouton number compared with controls (55.9 \pm 1.5 vs $37.0 \pm 0.8$, respectively) (Fig. $4 D, E, M$ ). This difference in bouton number continues through $264 \mathrm{~h} \mathrm{AEL}$, indicating that Hiw restricts NMJ growth during the ETI stage as it does during the period of normal larval development. After $240 \mathrm{~h}$ AEL, there is no further NMJ growth in hiw ${ }^{N D 8} /+$; phmGal4>UAS-torso-RNAi larvae and the NMJ growth curves for larvae with one or two copies of wild-type hiw converge at $288 \mathrm{~h}$ $\operatorname{AEL}(70.8 \pm 2.4$ and $69.7 \pm 2.8$, respectively). The basis of this convergence is unknown but could indicate that an upper limit for the maximum possible number of boutons is eventually reached or that Hiw ceases to have an important role in regulating NMJ growth around this time.

We also examined the role of wit during the ETI stage in phm-Gal4>UAS-torso-RNAi larvae. Between 84 and $144 \mathrm{~h} \mathrm{AEL,}$ NMJs in phm-Gal4>UAS-torso-RNAi larvae with one versus two copies of wild-type wit do not differ in bouton number (Fig. $4 A, C, N)$. However, beginning at $156 \mathrm{~h} \mathrm{AEL}$, bouton number is reduced in phm-Gal4>UAS-torso-RNAi larvae heterozygous for wit $(26.2 \pm 1.0$ vs $33.1 \pm 0.9)$ (Fig. $4 A, C, N)$ and this decrease in bouton number persists through $288 \mathrm{~h} \mathrm{AEL} \mathrm{(40.1 \pm 1.8} \mathrm{vs} 64.2 \pm$ 2.6) (Fig. $4 J, L, N$ ). Thus, BMP signaling continues to act as an important positive regulator of NMJ growth throughout the ETI period as it does during normal larval development.

Together, the results for hiw and wit suggest that continued growth during the ETI stage in phm-Gal4>UAS-torso-RNAi larvae remains under the control of these two major regulatory pathways as it is during the normal period of larval development, and this is likely to be true also for other NMJ regulatory pathways not examined here.

\section{Synaptic structure is maintained throughout the ETI stage}

Larval development in phm-Gal4>UAS-torso-RNAi individuals lasts approximately twice as long as normal, effectively doubling the larval "life span." Although this situation offers potentially novel opportunities to investigate time-dependent mechanisms of aging and neuroprotection using the larval NMJ, it is important to determine whether synaptic integrity is maintained in these larvae for the duration of the ETI period. Because the protective mechanisms that normally act to ensure maintenance of synaptic structure and function would likely have evolved to operate over the length of normal larval life, it is possible that as the $\mathrm{NMJ}$ ages well beyond its normal duration, NMJ integrity could degrade with time, resulting in disorganization of synaptic proteins and/or disassembly of individual boutons at late time points.

We examined synaptic integrity throughout the ETI period by labeling NMJs of phm-Gal4>UAS-torso-RNAi larvae with antibodies to key proteins. Anti-Dlg (Discs large) antibodies were used to label postsynaptic structures (Zito et al., 1997) and antiDvGlut (vesicular glutamate transporter; Daniels et al., 2004) antibodies were used to label presynaptic structures (Fig. 5A-L). Retraction or disassembly of NMJ structures would be expected to manifest as the appearance of synaptic footprints (Eaton and Davis, 2003), with the loss of postsynaptic proteins, or the accumulation of presynaptic debris (Fuentes-Medel et al., 2009). Throughout the entire ETI period up to $288 \mathrm{~h} \mathrm{AEL} \mathrm{(Fig.} \mathrm{5I-L),}$ the association of presynaptic DvGlut and postsynaptic Dlg remains unaltered in $p h m$-Gal4 $>U A S$-torso-RNAi larvae as in control larvae (Fig. 5A-D) and phm-Gal4>torso-RNAi larvae at 144 h (Fig. $5 E-H)$. Despite careful examination of numerous NMJs, we find no evidence for the appearance of ghost bouton 

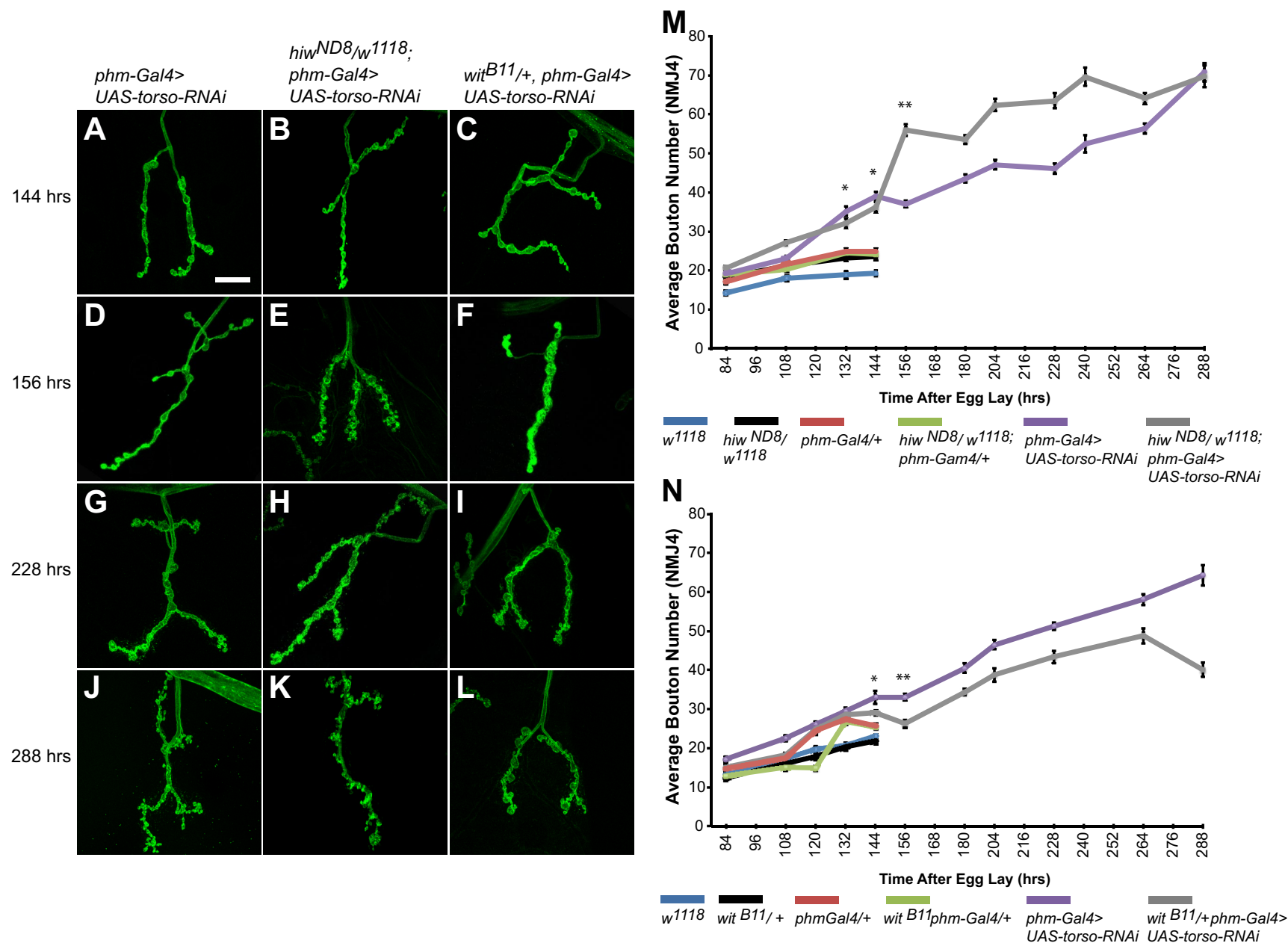

Figure 4. Synaptic growth during the ETI stage is sensitive to known regulators of synaptic growth. $A-L$, Confocal images of NMJ4 labeled with FITC-anti-HRP at selected time points during third instar. M, Quantification of average bouton number throughout third instar for $w^{1718}$ (blue line), hiw ${ }^{N D 8} / w^{1118}$ (black line), phm-Gal4/+ (red line), hiw ${ }^{N D 8} / w^{1118} ;$ phm-Gal4/+ (green line), phm-Gal4 > UAS-torso-RNAi (purple line), and hiw ${ }^{N D 8} / w^{1118} ;$ phm-Gal4 > UAS-torso-RNAi (gray line) larvae. $N$, Quantification of average bouton number throughout third instar for w ${ }^{1718}$ (blue line), wit $^{B 11} /+$ (black line), phm-Gal4/+ (red line), wit ${ }^{B 11}$ phm-Gal4/+ (green line), phm-Gal4>UAS-torso-RNAi (purple line), and wit ${ }^{B 11} /+$ phm-Gal4> UAS-torso-RNAi (gray line) larvae. Loss of one copy of the negative regulator hiw leads to a significant increase in average bouton number between 144 and $288 \mathrm{~h} \mathrm{AEL}(A, B, D, E, G, H, J, K, M)$. Loss of one copy of the positive regulator wit leads to a decrease in average bouton number between 144 and $288 \mathrm{~h}$ AEL $(\boldsymbol{A}, \mathbf{C}, \mathbf{D}, \boldsymbol{F}, \mathbf{G}, \mathbf{I}, \mathbf{J}, \mathbf{L}, \mathbf{N})$. Scale bar, $20 \mu \mathrm{m}$. Error bars represent SEM. ${ }^{*} p<0.01$ and ${ }^{* *} p<0.001$.

structures or postsynaptic footprints. Similarly, examination of synaptic microtubule organization (MT) using antibodies to the MT-associated protein Futsch (22C10), reveals no alterations in the MT cytoskeleton of phm-Gal4>UAS-torso-RNAi larvae at $288 \mathrm{~h}$ compared with control or phm-Gal4>UAS-torso-RNAi larvae at $144 \mathrm{~h}$ AEL controls (data not shown).

To assess formation and maintenance of properly apposed active zones and postsynaptic receptors, we labeled NMJs with antibodies to the common type III glutamate receptor subunit, GluRIII, and the essential active zone protein Bruchpilot, Brp (Fig. 5M-O). At $288 \mathrm{~h} \mathrm{AEL}$, boutons in phm-Gal4>UAS-torsoRNAi larvae appear larger and contain more active zones per bouton than normal (Fig. 5M-3,N-3,O-3; data not shown). However, close apposition of glutamate release sites and receptor fields is maintained. The appearance of unaltered presynaptic and postsynaptic apposition is not a result of projecting multiple optical slices, since a single $0.5 \mu \mathrm{m}$ section reveals proper apposition of Brp and GluRIII (Fig. 5M-4,N4,O-4). For active zones in the single-slice images that appear labeled by Brp antibody only, the corresponding postsynaptic GluRIII clustering is easily identified in adjoining optical slices. These results suggest that the overall appearance and organization of presynaptic and postsyn- aptic structures is maintained in phm-Gal4>UAS-torso-RNAi larvae throughout their expanded larval life.

\section{Synaptic function is unaffected during the ETI stage}

Despite the normal appearance of presynaptic and postsynaptic structures at NMJs in phm-Gal4>UAS torso-RNAi larvae, more subtle perturbations could accumulate over time resulting in defects in synaptic function. To examine this possibility, we monitored NMJ function over time by recording both spontaneous and evoked transmitter release in phm-Gal4>UAS torso-RNAi larvae (Fig. 6) throughout the ETI period. At $132 \mathrm{~h} \mathrm{AEL}$, we find no difference in phm-Gal4>UAS torso-RNAi larvae compared with controls in amplitude of EJPs (Fig. 6A-F,O), amplitude of spontaneous mEJPs (Fig. $6 G-L, M$ ), frequency of mEJPs (Fig. $6 G-L, N$ ), or in quantal content (Fig. 6P). Moreover, these parameters do not change significantly over time in $\mathrm{phm}$ Gal4>UAS-torso-RNAi larvae for the entire duration of the ETI period (Fig. 6). These data strongly suggest that NMJ function, like NMJ structure, remains essentially normal in phm-Gal4> UAS torso-RNAi larvae despite the fact that these synapses persist twice as long as in control larvae. 

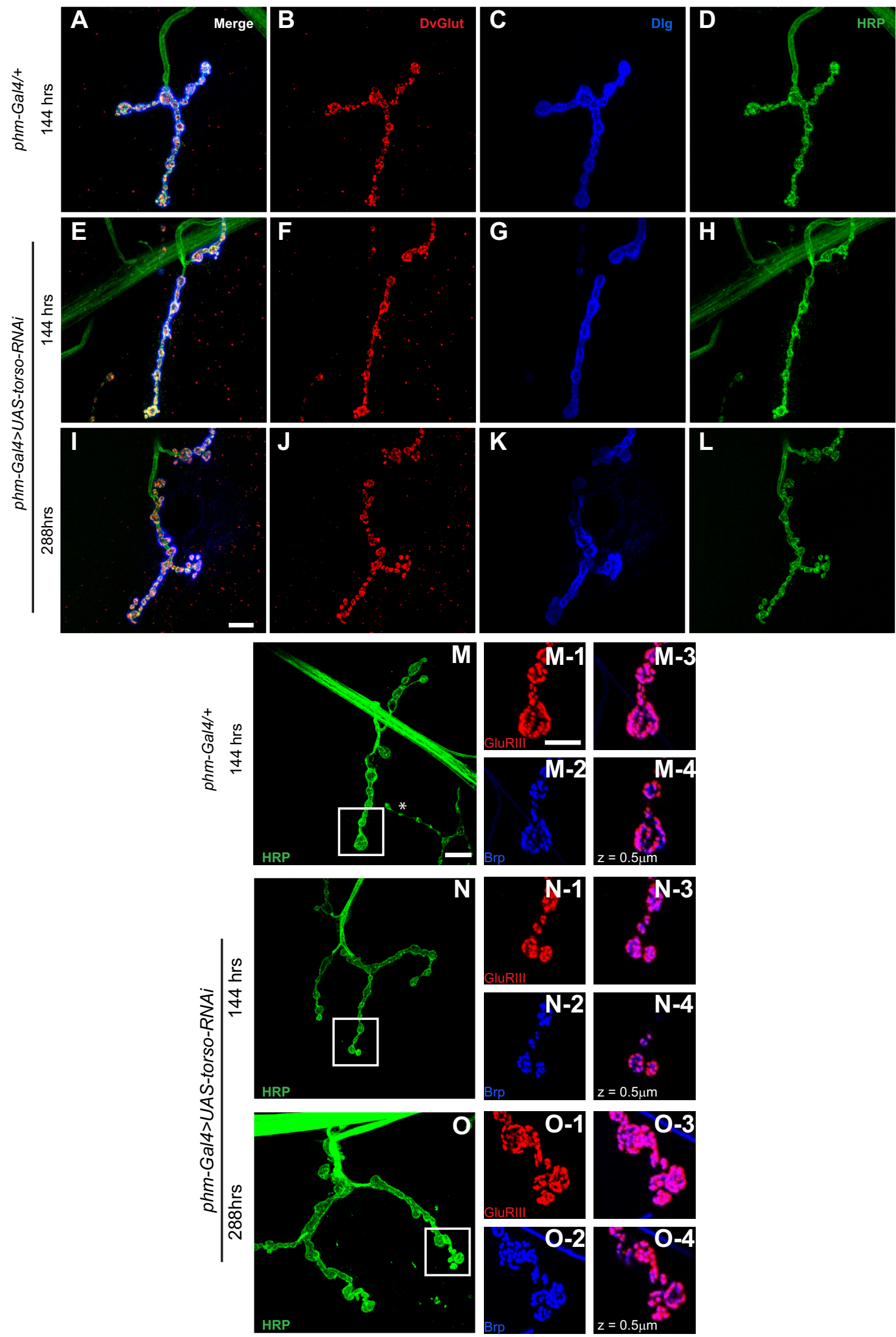

Figure 5. Synaptic structure and protein localization remain normal throughout the ETI stage. $A-L$, Maximum intensity projections of confocal images of NMJ4 labeled with anti-DvGlut $(\boldsymbol{B}, F, \boldsymbol{J})$, anti-Dlg $(\boldsymbol{C}, \boldsymbol{G}, \boldsymbol{K})$, and anti-HRP $(\boldsymbol{D}, \boldsymbol{H}, \boldsymbol{L})$. Genotypes and age of larvae at time of dissection are indicated. $\boldsymbol{M}-\mathbf{0}$, High-magnification images of NMJ 4 terminal boutons labeled with anti-GluRIII ( $\boldsymbol{M}$ - $\mathbf{1}$, $\mathbf{N}-\mathbf{1}, \mathbf{0 - 1})$ and anti-Brp $(\boldsymbol{M}-\mathbf{2}, \mathbf{N}-\mathbf{2}, \mathbf{0 - 2})$. All panels show maximum intensity projections of consecutive $0.4 \mu \mathrm{m}(\boldsymbol{A}-\mathbf{L})$ or $0.5 \mu \mathrm{m}(\boldsymbol{M}-\mathbf{0})$ sections, except for $\boldsymbol{M}-\mathbf{4}, \mathbf{N}-\mathbf{4}$, and $\mathbf{0 - 4}$, which show a single $0.5 \mu \mathrm{m}$ optical section. $\mathbf{M}-\mathbf{3}, \mathbf{N}-\mathbf{3}$, and $\mathbf{0 - 3}$ show merged images of GluRlll and Brp labeling. Scale bars, $10 \mu \mathrm{m}$. Asterisk indicates type Is NMJ on muscle 4. 

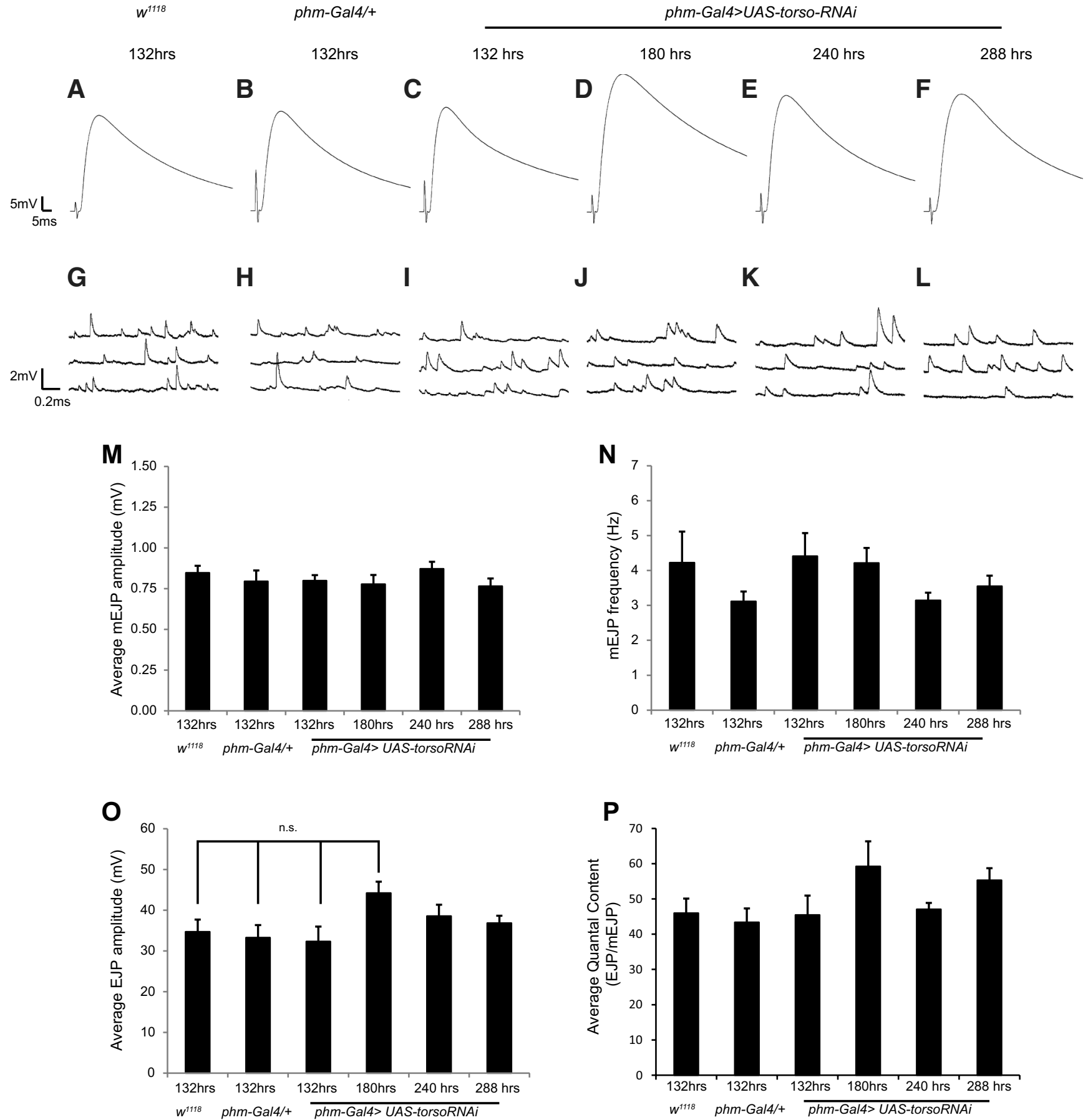

Figure 6. Larval NMJs remain functionally normal throughout the ETI stage. $\boldsymbol{A}-\boldsymbol{F}$, Representative traces of EJPs from third instar larvae of the indicated genotypes and time points after egg lay. $\boldsymbol{G}-\boldsymbol{L}$, Representative traces of spontaneous neurotransmitter release (mEJPs) from third instar larvae of the indicated genotypes and time points AEL. All recordings $(\boldsymbol{A}-\boldsymbol{L})$ were acquired in $1.0 \mathrm{mM}$ $\mathrm{Ca}^{2+} . \boldsymbol{M}-\boldsymbol{P}$, Quantification of average mEJP amplitude $(\boldsymbol{M})$, average mEJP frequency $(\boldsymbol{M})$, average EJP amplitude $(\boldsymbol{O})$, and average quantal content $(\boldsymbol{P})$. Error bars represent SEM. n.s. $=$ not statistically significant.

\section{Discussion}

The Drosophila larval NMJ is a powerful system for studying many neurobiological problems including genetic and molecular analyses of neuronal excitability, synaptic transmission, and synaptic development. However, it has not been used extensively for studying age-dependent processes such as maintenance of neuronal viability and synaptic stability. This is because of limitations imposed by the onset of pupariation 2-3 d after larvae enter the third instar and the consequent remodeling of the larval nervous system. Here, we circumvent this limitation by taking advantage of phm-Gal4>UAS-torso-RNAi larvae, which undergo largely normal first and second larval instars but have a significantly expanded third instar (Rewitz et al., 2009). This expansion raises interesting questions about neuronal and synaptic viability and maintenance with age because phm-Gal4 $>U A S$-torso-RNAi larvae persist well beyond the normal time over which normal larval neuroprotective mechanisms have been selected to operate. Do the mechanisms that maintain proper synaptic growth, structure, and 
function continue to operate over the course of the ETI? Our characterization of synaptic growth, structure, and function in phmGal4>UAS-torso-RNAi larvae establishes the foundation for their future use in modeling motor neuron diseases and for investigating time-dependent effects of perturbing normal mechanisms of neuroprotection, synaptic maintenance, and response to neural injury.

\section{Dynamics of NMJ growth in phm-Gal4> UAS-torso-RNAi larvae}

Larval NMJ growth terminates at pupariation, $\sim 144 \mathrm{~h}$ AEL in control larvae. However, in phm-Gal4>UAS-torso-RNAi larvae, NMJ4 maintains constant growth over the ETI, resulting in a threefold increase in boutons compared with control larvae. Thus, NMJ growth mechanisms continue to operate until $288 \mathrm{~h}$ AEL when phm-Gal4>UAS-torso-RNAi larvae finally pupariate, suggesting that NMJ growth is not normally constrained by termination of growth-promoting pathways or by achieving some predetermined maximal size.

NMJs normally increase in size throughout larval development roughly in parallel with muscle growth. This parallel growth is evident in phm-Gal4>UAS-torso-RNAi larvae until $\sim 180 \mathrm{~h}$ AEL. However, starting between 180 and $204 \mathrm{~h} \mathrm{AEL}$, the rates of muscle growth and bouton addition diverge; NMJs continue to grow at an unchanged rate, whereas muscle growth levels off. Thus, although growth of muscles and NMJs occurs in concert during much of normal larval development and throughout early stages in phm-Gal4>UAS-torso-RNAi larvae, during later stages of the ETI period, they are uncoupled. Various mutants affecting NMJ growth are often described in terms of boutons/ muscle surface area. Although this normalization may sometimes be appropriate, it is important to recognize that the number of boutons per NMJ size is not strictly dependent on muscle surface area and that different mutants could affect these two processes in distinct ways.

The basic NMJ branching pattern is established early and does not change significantly throughout normal larval development (Zito et al., 1999). NMJ growth in phm-Gal4>UAS-torso-RNAi larvae largely follows the same pattern. Even though the number of boutons at NMJ4 is threefold larger by $288 \mathrm{~h} \mathrm{AEL}$, on average only one new branch is added between 144 and $288 \mathrm{~h}$ AEL. These results suggest that various aspects of NMJ architecture are regulated separately and respond differently to an extended growth period. Whereas addition of new boutons is plastic and occurs at a constant rate throughout the ETI, the number of synaptic branches is established early and is relatively stable thereafter. Similarly, linear extension of synaptic branches stops in phmGal4>UAS-torso-RNAi larvae $\sim 144 \mathrm{~h} \mathrm{AEL}$, the time when control larvae undergo pupariation. This suggests that linear growth may be temporally fixed even when larval development is extended. Since addition of new boutons continues unabated, the net result is increased bouton density along synaptic branches at late stages in phm-Gal4>UAS-torso-RNAi larvae. These results also indicate that much of the increase in bouton number in phm-Gal4>UAS-torso-RNAi larvae between 144 and $288 \mathrm{~h}$ AEL is by interstitial addition of new boutons rather than at growing termini. The appearance of satellite boutons and hyperbudded boutons in later stages of the ETI can be similarly explained.

\section{Ecdysone signaling limits NMJ growth}

Unexpectedly, bouton number in phm-Gal4>UAS-torso-RNAi larvae already diverges from controls by $132 \mathrm{~h}$ AEL. How can phm-Gal4>UAS-torso-RNAi larvae anticipate the extended period of NMJ growth, which should nominally only begin $\sim 144 \mathrm{~h}$ AEL? Reduction of torso transcripts in muscles or motor neurons via $U A S$-torso-RNAi has no effect, indicating that the early increase in bouton number is not directly dependent on perturbed torso signaling in these cells. However, reduced torso expression in the PG causes systemic reduction in ecdysone signaling, which could affect NMJ growth. Reduction of ecdysone receptor isoforms in muscle using RNAi does not affect bouton number, but similar knockdown in motor neurons causes an increase in both bouton number and muscle area by $120 \mathrm{~h}$ AEL. Thus, ecdysone appears to act directly in motor neurons to limit NMJ growth. Perhaps ecdysone-dependent restraint of NMJ growth at this stage is a prelude to the extensive remodeling of motor neurons that, during normal development, will begin shortly thereafter with the onset of metamorphosis.

\section{Regulators of NMJ growth in phm-Gal4>UAS-torso-RNAi larvae}

The inferences drawn above about NMJ growth dynamics as well as about the role of ecdysone signaling, illustrate the kind of useful new information that can be gleaned from examining extended NMJ growth in phm-Gal4>UAS-torso-RNAi larvae. It would have been difficult to obtain these insights from studies of normal NMJ development. Nonetheless, it is important to emphasize that although NMJs in phm-Gal4>UAS-torso-RNAi larvae differ from normal, these differences appear to be the inevitable result of extending the time over which NMJ growth occurs. In contrast with various mutants that perturb NMJ development and result in aberrant patterns of NMJ growth, the NMJ phenotypes of phm-Gal4>UAS-torso-RNAi larvae can be considered to result from an extension of normal growth mechanisms, i.e., the mechanisms that normally regulate NMJ growth continue to operate but do so over an aberrant time span.

Consistent with the idea that NMJ growth is regulated normally in phm-Gal4>UAS-torso-RNAi larvae, the NMJ phenotype does not differ from control larvae until $132 \mathrm{~h}$ AEL when bouton number in phm-Gal4>UAS-torso-RNAi larvae continues to increase while it levels off before pupariation in control larvae. Subsequently, further NMJ growth in phm-Gal4>UAS-torso-RNAi larvae is regulated by the same factors that act earlier during normal NMJ development. In particular, heterozygosity for the negative regulator, hiw, leads to a further increase in bouton number, while heterozygosity for the positive regulator, wit, causes a decrease in bouton number. Heterozygosity for either of these mutations has no effect on NMJ growth in wild-type larvae or in phm-Gal4>UAS-torso-RNAi larvae before $156 \mathrm{~h}$ AEL. Thus, NMJ growth in phm-Gal4>UAS-torso-RNAi larvae not only remains dependent on normal regulators throughout the ETI, but in addition, this growth is even more sensitive than normal to loss of these regulators. The basis of this enhanced sensitivity is unknown, but the fact that it is manifest only at later stages of the ETI could mean that wit and hiw are downregulated in wild-type larvae around the time pupariation begins. If this downregulation occurs at the same chronological time in phm-Gal4>UAS-torsoRNAi larvae, a further $50 \%$ reduction in expression because of heterozygosity for hiw or wit mutations could decrease Hiw and Wit levels below some critical threshold resulting in the observed phenotypes. Dependence of NMJ growth in phm-Gal4>UAS-torso-RNAi larvae on Wit throughout the ETI is of further interest because it shows that BMP signaling is not just a permissive trigger that initiates $\mathrm{NMJ}$ growth but is required continuously to sustain NMJ growth.

\section{Synaptic structure and function are maintained in phm-Gal4>UAS-torso-RNAi larvae}

A major impetus for these studies was to develop an experimental model that uses the larval NMJ for studies of time-dependent processes such as neurodegeneration and synaptic destabilization. The 
extension of the larval stage in phm-Gal4>UAS-torso-RNAi larvae seems to provide the time window needed to achieve this aim. However, this very feature of $p h m$-Gal4 $>U A S$-torso-RNA $i$ larvae was itself of possible concern because of uncertainty whether NMJs would remain fully intact and functional when the larval period was doubled. If not, use of these larvae to study various perturbations for their effects on long-term synaptic maintenance and function would be limited. However, we found no indication that NMJs in phmGal4>UAS-torso-RNAi larvae retract or become destabilized over extended larval life. Not only did the overall appearance of the NMJs remain robust and stable, but also proper synaptic organization was maintained as revealed by antibody labeling of various presynaptic and postsynaptic components. Maintenance of NMJ structural integrity in phm-Gal4>UAS-torso-RNAi larvae is paralleled by sustained functional integrity as well; there is no evidence for a decrement in synaptic transmission during extended larval life.

Despite the increase in bouton number throughout the ETI period, the EJP amplitude remains nearly constant. This lack of correlation between bouton number and amount of transmitter release is consistent with recent quantal analysis of synaptic transmission in normal larvae (Guerrero et al., 2005; Peled and Isacoff, 2011). These studies revealed that approximately half of the presynaptic active zones have little or no transmitter release in response to nerve stimulation and that only $10 \%$ of release sites have release probabilities $>0.2$. Thus, only a small number of release sites dominate basal transmission at the NMJ and these sites are preferentially localized in terminal boutons (Guerrero et al., 2005; Peled and Isacoff, 2011). These observations can account for the disparity between bouton numbers and EJP size previously observed in various mutants (Coyle et al., 2004; Collins et al., 2006; Seabrooke and Stewart, 2008) as well as in different species of Drosophila (Campbell and Ganetzky, 2012). Presumably, the same mechanisms that regulate release probability and determine the location of high probability release sites contribute to the stability of total release in ETI larvae as their NMJs continue to expand by addition of new boutons. Consequently, despite their persistence over a much longer life span, NMJs in phmGal4 $>U A S$-torso-RNA $i$ larvae remain essentially normal.

phm-Gal4>UAS-torso-RNAi larvae as an experimental model In conclusion, our studies demonstrate that the features that make the larval NMJ such a powerful system for neurobiological studies are largely preserved in phm-Gal4 $>U A S$-torso-RNAi larvae, even though the duration of the third instar period is extended threefold. Use of these larvae should thus enable future studies to probe time-dependent neurobiological processes such as synaptic degeneration in motor neuron disease, which have important implications for human neurological disorders.

\section{References}

Aberle H, Haghighi AP, Fetter RD, McCabe BD, Magalhães TR, Goodman CS (2002) wishful thinking encodes a BMP type II receptor that regulates synaptic growth in drosophila. Neuron 33:545-558.

Budnik V, Zhong Y, Wu CF (1990) Morphological plasticity of motor axons in Drosophila mutants with altered excitability. J Neurosci 10:3754-3768.

Budnik V, Koh YH, Guan B, Hartmann B, Hough C, Woods D, Gorczyca M (1996) Regulation of synapse structure and function by the Drosophila tumor suppressor gene dlg. Neuron 17:627-640.

Campbell M, Ganetzky B (2012) Extensive morphological divergence and rapid evolution of the larval neuromuscular junction in Drosophila. Proc Natl Acad Sci U S A 109:E648-E655.

Collins CA, DiAntonio A (2007) Synaptic development: insights from Drosophila. Curr Opin Neurobiol 17:35-42.

Collins CA, Wairkar YP, Johnson SL, DiAntonio A (2006) Highwire re- strains synaptic growth by attenuating a MAP kinase signal. Neuron 51:57-69.

Coyle IP, Koh YH, Lee WC, Slind J, Fergestad T, Littleton JT, Ganetzky B (2004) Nervous wreck, an SH3 adaptor protein that interacts with Wsp, regulates synaptic growth in Drosophila. Neuron 41:521-534.

Daniels RW, Collins CA, Gelfand MV, Dant J, Brooks ES, Krantz DE, DiAntonio A (2004) Increased expression of the Drosophila vesicular glutamate transporter leads to excess glutamate release and a compensatory decrease in quantal content. J Neurosci 24:10466-10474.

Dickman DK, Lu Z, Meinertzhagen IA, Schwarz TL (2006) Altered synaptic development and active zone spacing in endocytosis mutants. Curr Biol 16:591-598.

Eaton BA, Davis GW (2003) Synapse disassembly. Genes Dev 17:2075-2082.

Featherstone DE, Broadie K (2000) Surprises from Drosophila: genetic mechanisms of synaptic development and plasticity. Brain Res Bull 53:501-511.

Fuentes-Medel Y, Logan MA, Ashley J, Ataman B, Budnik V, Freeman MR (2009) Glia and muscle sculpt neuromuscular arbors by engulfing destabilized synaptic boutons and shed presynaptic debris. PLoS Biol 7:e1000184.

Gilbert LI, Rybczynski R, Warren JT (2002) Control and biochemical nature of the ecdysteroidogenic pathway. Annu Rev Entomol 47:883-916.

Guan B, Hartmann B, Kho YH, Gorczyca M, Budnik V (1996) The Drosophila tumor suppressor gene, dlg, is involved in structural plasticity at a glutamatergic synapse. Curr Biol 6:695-706.

Guerrero G, Reiff DF, Agarwal G, Ball RW, Borst A, Goodman CS, Isacoff EY (2005) Heterogeneity in synaptic transmission along a Drosophila larval motor axon. Nat Neurosci 8:1188-1196.

Jan LY, Jan YN (1976) Properties of the larval neuromuscular junction in Drosophila melanogaster. J Physiol 262:189-214.

Keshishian H, Broadie K, Chiba A, Bate M (1996) The Drosophila neuromuscular junction: a model system for studying synaptic development and function. Annu Rev Neurosci 19:545-575.

Marqués G (2005) Morphogens and synaptogenesis in Drosophila. J Neurobiol 64:417-434.

Marqués G, Bao H, Haerry TE, Shimell MJ, Duchek P, Zhang B, O’Connor MB (2002) The Drosophila BMP type II receptor Wishful Thinking regulates neuromuscular synapse morphology and function. Neuron 33:529-543.

McBrayer Z, Ono H, Shimell M, Parvy JP, Beckstead RB, Warren JT, Thummel CS, Dauphin-Villemant C, Gilbert LI, O'Connor MB (2007) Prothoracicotropic hormone regulates developmental timing and body size in Drosophila. Dev Cell 13:857-871.

McLachlan EM, Martin AR (1981) Non-linear summation of end-plate potentials in the frog and mouse. J Physiol 311:307-324.

Packard M, Mathew D, Budnik V (2003) FASt remodeling of synapses in Drosophila. Curr Opin Neurobiol 13:527-534.

Peled ES, Isacoff EY (2011) Optical quantal analysis of synaptic transmission in wild-type and rab3-mutant Drosophila motor axons. Nat Neurosci 14:519-526.

Rewitz KF, Yamanaka N, Gilbert LI, O'Connor MB (2009) The insect neuropeptide PTTH activates receptor tyrosine kinase torso to initiate metamorphosis. Science 326:1403-1405.

Ruiz-Cañada C, Budnik V (2006) Introduction on the use of the Drosophila embryonic/larval neuromuscular junction as a model system to study synapse development and function, and a brief summary of pathfinding and target recognition. Int Rev Neurobiol 75:1-31.

Seabrooke S, Stewart BA (2008) Moesin helps to restrain synaptic growth at the Drosophila neuromuscular junction. Dev Neurobiol 68:379-391.

Shen W, Ganetzky B (2009) Autophagy promotes synapse development in Drosophila. J Cell Biol 187:71-79.

Wan HI, DiAntonio A, Fetter RD, Bergstrom K, Strauss R, Goodman CS (2000) Highwire regulates synaptic growth in Drosophila. Neuron 26:313-329.

Zito K, Fetter RD, Goodman CS, Isacoff EY (1997) Synaptic clustering of Fascilin II and Shaker: essential targeting sequences and role of Dlg. Neuron 19:1007-1016.

Zito K, Parnas D, Fetter RD, Isacoff EY, Goodman CS (1999) Watching a synapse grow: noninvasive confocal imaging of synaptic growth in Drosophila. Neuron 22:719-729. 